\title{
Electroencephalographic characteristics of attention-deficit/hyperactivity disorder subtypes
}

\author{
Mohammad Rostami', Reza Khosrowabadi',** (D, Hamidreza Pouretemad ${ }^{3}$
}

\begin{abstract}
1. PhD Student of Cognitive Psychology, Institute for Cognitive and Brain Sciences, Shahid Beheshti University, Tehran, Iran
2. Assistant Professor of Cognitive Modeling, Institute for Cognitive and Brain Sciences, Shahid Beheshti University, Tehran, Iran

3. Professor of Cognitive Psychology Department, Institute for Cognitive and Brain Sciences, Shahid Beheshti University, Tehran, Iran
\end{abstract}

Recieved: . 6 May 2019

Revised: . 16 Jun. 2019

Accepted: 4 Jul. 2019

\section{Keywords}

Electroencephalography (EEG) Attention deficit disorder with hyperactivity subtypes

Additive Model

\section{Corresponding author}

Reza Khosrowabadi, Assistant Professor of Cognitive Modeling, Institute for Cognitive and Brain Science, Shahid Beheshti University, Evin Sq, Tehran, Iran

Email: R_khosrowabadi@sbu.ac.ir

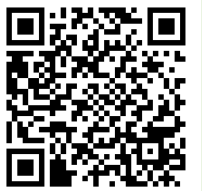

\section{Abstract}

Introduction: Attention-Deficit/Hyperactivity Disorder (ADHD as a neurodevelopmental disorder includes three subtypes predominantly inattentive (ADHD-I), predominantly impulsive/hyperactive (ADHD-H), and predominantly combined (ADHD-C) that has formed a heterogeneity disorder due to differences in pathology and syndrome. This study aimed to evaluate the Electroencephalographic (EEG) characteristics of ADHD subtypes.

Methods: Subjects included 61 boys (7-12 years old, normal intelligence) with ADHD (ADHD-I, N=25; ADHD-H, N=14 and ADHD-C, N=22) and 43 typically developing children (TDC). The groups were matched for IQ- and age. Children were investigated regarding Child Behavior Checklist and brain electrical activity during eyes-closed resting state (i.e. EEG power in delta (1-4 Hz), Theta (4-8 Hz), Alpha (8-12 Hz) and Beta $(12-30 \mathrm{~Hz})$ frequency bands. Statistical analysis included a $2 \times 2$ factorial design by the additive model.

Results: Theta/Beta ratio was higher in all ADHD subtypes, especially the ADHD-I subtypes, compared to normal children. Besides, in the ADHD-I subtype, theta activity was higher and beta activity was lower than Theta. No significant difference found between the groups in delta and alpha activity.

Conclusion: According to these results, EEG markers can better distinguish the ADHD-I subtype, which can be explained within the framework of the maturational lag model of the central nervous system. 


\title{
مشخصات الكتروانسفالوكر افى زير كروههاى كودكان با نقص توجه/بيشفعالى
}

\author{
محمد رستمىى'، رضا خسرو آبادى ז"iD) ، حميدرضا يوراعتمادץ

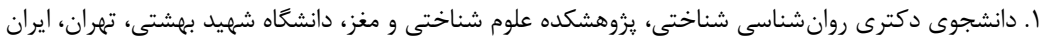

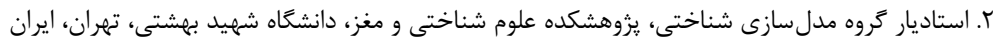

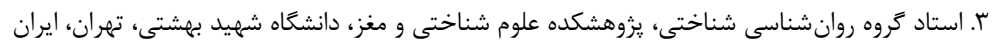

\section{ars}

مقلدمه: اختلال نقص توجه/بيشفعالى به عنوان يك اختلال عصب تحولى شامل سه زير گروه نقص توجه، بيشفعالى| تكانشگرى و تركيبى است كه با توجه به تفاوت در سبب شناسى و نشانهشناسى اختلال ناهمخنى را شكل مىدهد. هدف اين مطالعه بررسى مشخصات الكتروانسفالوكرافى در زير كروههاى اختلال نقص توجه/بيشفعالى بود. روش كار: آزمودنى هاى اين مطالعه را او يسر مبتلا به اختلال نقص توجه/بيشفعالى (زير كروه نقص توجه: هץ نفر؛ زير

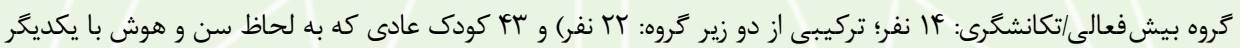
همتا شده بودند، تشكليل دادند. آزمودنى ها به وسيله سياهه رفتارى كودكان و الكتروانسفالوكرافى (دلتا: ا تا \& هرتز، تتا:

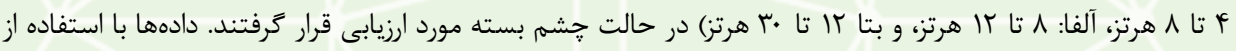
تحليل عاملى دو راهه و به وسيله مدل افزايشى و با نرمافزار SPSS تجزيه و تحليل شدند. يافته ها: نسبت تتا/بتا در همه زير كروههاى اختلال نقص توجه/بيشفعالى به ويثه زير كروه نقص توجه در مقايسه با كودكان عادى بالاتر بود. به علاوه در زير گروه نقص توجه، فعاليت تتا بالاتر و فعاليت بتا پايينتر بود. هيج تفاوت معنادارى بين كروهها در فعاليت دلتا و آلفا مشاهده نشد. نتيجه گَيرى: بر اساس نتايج اين مطالعه، نشانكرهاى الكتروانسفالوكرافى زير گروه نقص توجه را بهتر تشخيص مى دهند كه اين مىتواند در خارجوب مدل تاخير بلوغ سيسته اعصاب مركزى تبيين گردد.
\end{abstract}

دريافت:

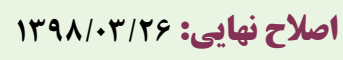

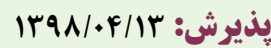

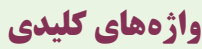
الكتروانسفالو اختلال نقص توجه/بيشفعالى

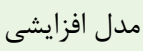

نويسنله مسئول رضا خسروآبادى، استاديار تروه مدل سازى شناختى، يزوهشكده علوم شناختى و مغز، دانشكاه شهيد بهشتى، ميدان اوين، تهروان

ايميل:Rhosrowabadi@sbu.ac.ir

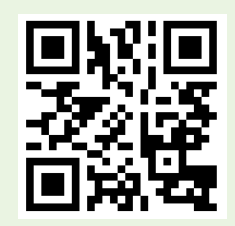

dol doi.org/10.30699/icss.22.1.70
ميزان شيوع آن در ايران حدود • ا درصد ززارش شده است (؟). اين اختلال داراى سه زير گروه نقص توجه (Inattention (ADHD-I))، بيشفعالى/تكانشخرى (Hyperactivity-impulsivity (ADHD-H)) و نوع تركيبى (Combined ADHD-C) است. مطالعات مختلفى اشاره كردهاند كه ADHD اختلال واحد و متجانسى نيست و زير گروههاى آن peractivity Disorder ((ADHD) اختلال نقص توجه/بيشفعالى يك اختلال بسيار شايع است كه جزء اختلالات Attention deficit/Hhy روان يزشكى كودكان و نوجوانان در نظر گرفته مىشود. بر اساس ملاك راهنماى تشخيصى و آمارى اختلالات روانى ميزان شيوع اين اختلال در كودكان ه درصد و در بزركسالان ه/ T درصد مىباشد ( (1)، همجنين 
TBR حمايت نكردند (ها - • (). مطالعات فراتحليل، افزايش ADHD

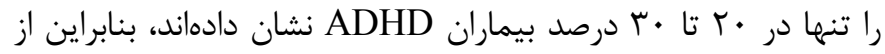
نظر اين يروهشكَران TBR نمىتواند به عنوان يك نشانكر تشخيصى يايا براى تشخيص ADHD در نظر ترفته شود. (ه|). يكى از مهمترين

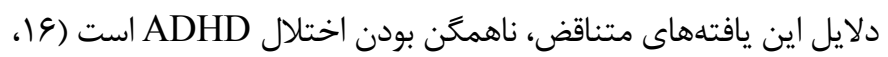
IV

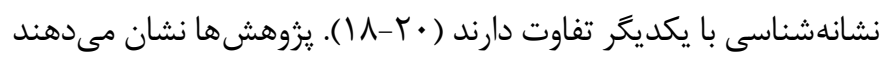
كه با افزايش سن فعاليت EEG افراد ADHD-I و ADHD-C مشابه هم است. در همين راستا Clarck و همكاران معتقدند كه علائم زير كروه

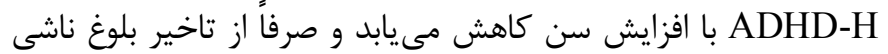

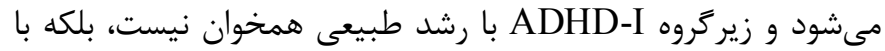
انحراف دائمى رشدى در كاركرد سيستم عصبى مركزى رابطه دارد كه ممكن است اين تفاوت در فعاليت الكتريكى مغز نيز نمود يابد (ع) (1).

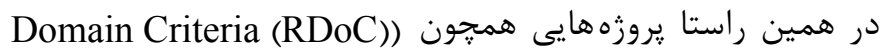

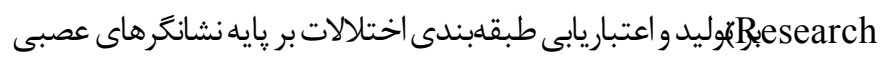
تاكيد دارند (IT). براى فهم بهتر اين مسئله لازم است كه مطالعاتى بر روى تفاوت نتايج EEG در زيركروههاى ADHD انجام گيرد. به همين علت اين مطالعه با هدف مقايسه الكَوهاى الكتروانسفالوَّرافى كمّى اين كودكان بر اساس زيركروههاى آن انجام كرفت.

\section{روش كار}

اين يزوهش از نوع نيمه آزمايشى بود. تعداد الو كودى ADHD (نوع

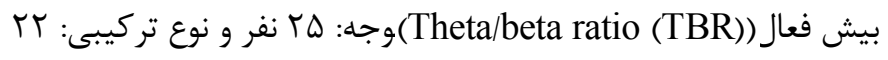

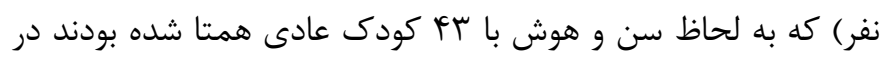

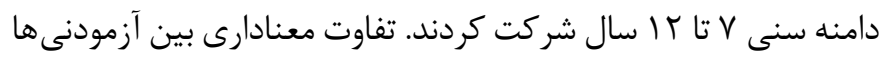
در سن و هوش مشاهده نشد (جدول (). كودكان ADHD به روش نمونه كيرى در دسترس از درمانكاههاى روان يزشكى شهر تهران انتخاب شدند. تشخيص كودكان ADHD بر اساس مصاحبه بالينى ساختاريافته بركرفته (Structured Clinical Interview for DSM-V (SCID)) شده از نسخه فارسى DSM-V توسط روانيزشك و روانشناس انجام

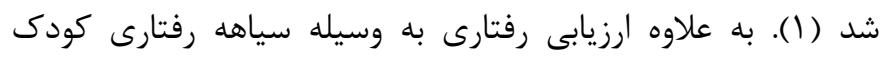

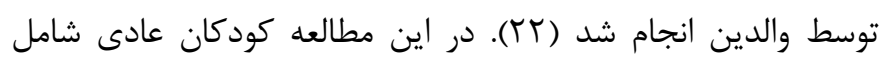

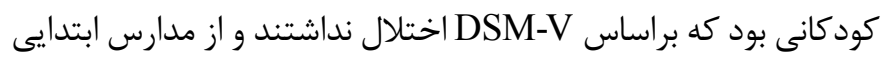

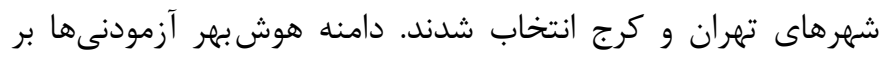

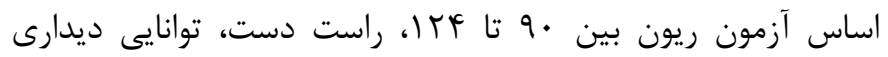
و شنيدارى طبيعى بودند. ملاك خروج از مطالعه شامل تاريخجه

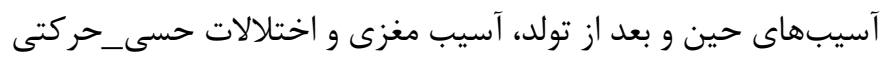

به لحاظ سببشناسى، نشانهشناسى، نوع و ياسخ درمان، سن شروع، شدت اختلال، ميزان شيوع و همايندى با ديكر اختلالات با يكديكر متفاوت

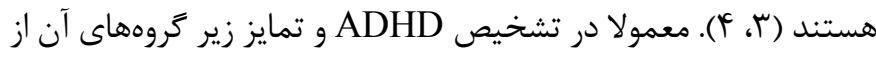
روشهاى معمول مانند مصاحبه با بيمار و والدين و اغلب از آزمون هاى

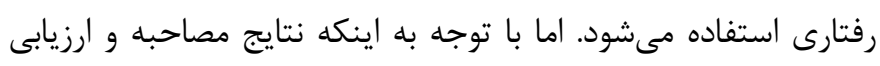

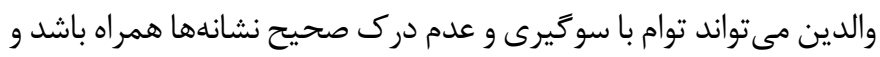

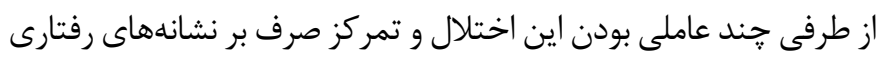
مانع از تشخيص صحيح و باياى اين اختلال مى شئدود. با توجه به اينكه ADHDبا آسيبهاى نوروفيزيولوزيكى رابطه دارد، بنابراين روشهاى نوروفيزيولوزيكى مىتوانند در تشخيص اين اختلال كمك كنند.

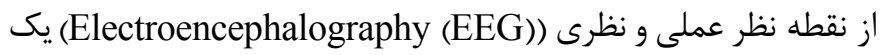
ابزار كمك تشخيصى ايدهآل براى اختلالات عصب تحولى است. EEG

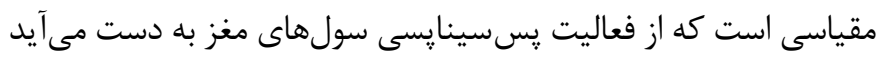

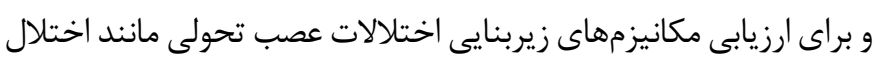

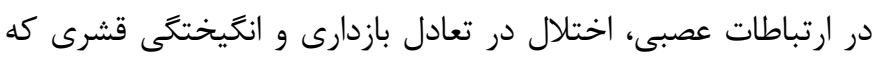

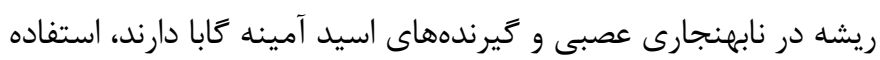

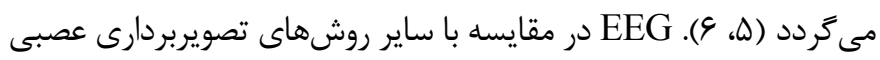
مانند fMRI از دقت زمانى بسيار بالا، سهولت اجرا، هزينه كمتر و حساسيت

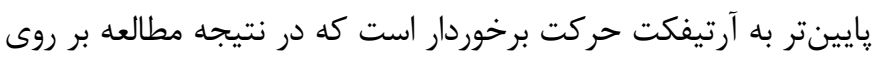

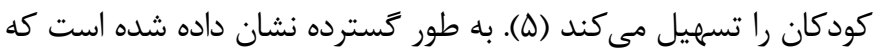

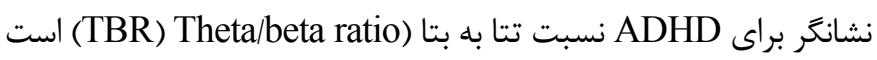

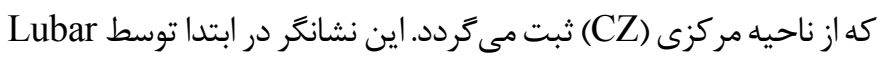

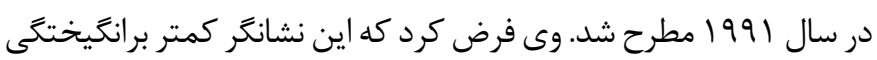

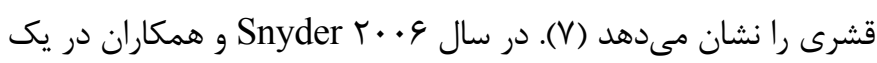

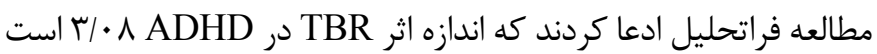

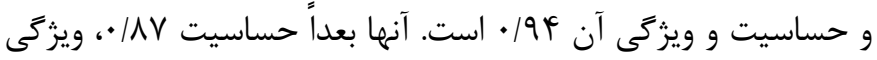

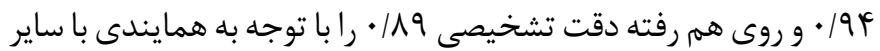
اختلالات روان يزشكى، سطح رشد (كودى، نوجوان)، جنسيت و كروه

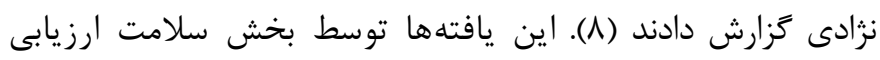

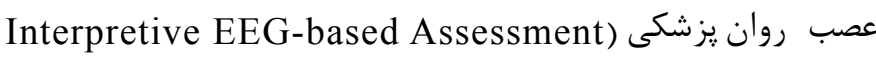

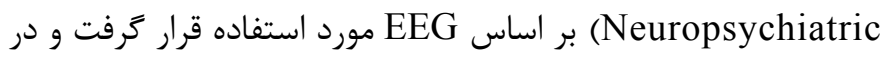
جولاى سال با • ب به عنوان روش كمك تشخيصى توسط سازمان غذا

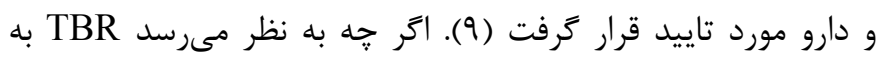
عنوان نشانكر تشخيصى موفقيت آميز است اما اختلاف نظر در اين مورد الند همجنان ادامه دارد. اين اختلاف نظر از مجموعه مطالعاتى ناشى مىشود كه توسط كروههاى ارد ادرى

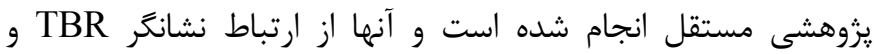


عمل را برق نكار مغزى (EEG) مى گويند. به علاوه اين امواج پيس از ترسيم مىتوانند مورد تجزيه و تحليل قرار كيرند و به شكل كمى در بيايند، كه اين تحليل كمى از امواج مغزى ثبت شده را برق نغار مغزى

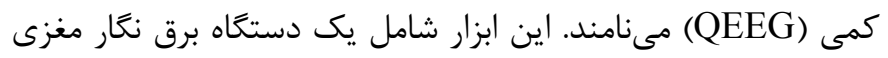

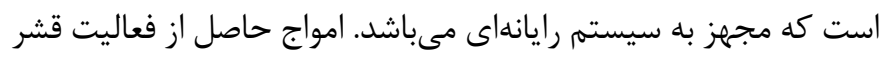

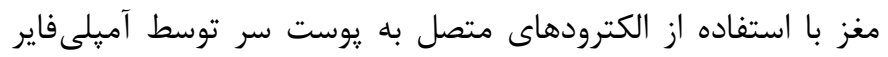

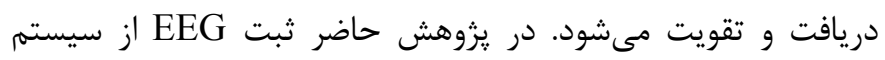
در حالت جشم بسته انجام شد و براى تحليل دادهها از برنامه MATLAB و بسته نرمافزارى EEGLAB استفاده شد.

\section{روش تجزيه و تحليل آمارى دادهها} با استفاده از مدل افزايشى (Additive Model) (شكل ()) و تحليل

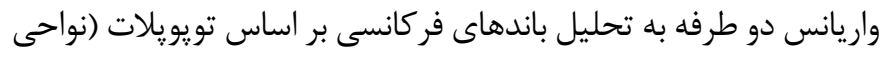
مغزى) يرداخته شد، كه در آن نواحى ساجيتال (Sagittal) (فرونتال:

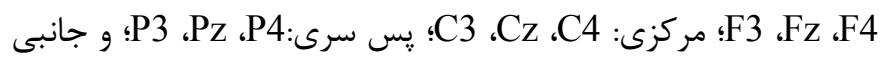

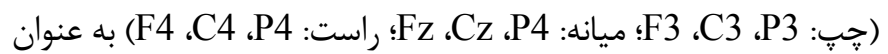
عامل درون گروهى و مشكلات بيشفعالى/تكانشكرى و نقص توجه به عنوان عامل بين گروهى در نظر گرفته شد. تحليل دادهها با استفاده از

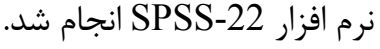

\section{a) Hyperactivity / Impulsivity}

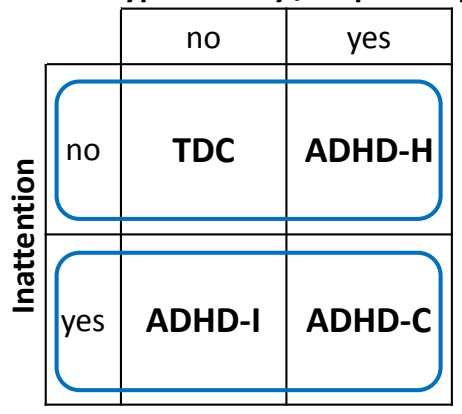

C) Hyperactivity / Impulsivity

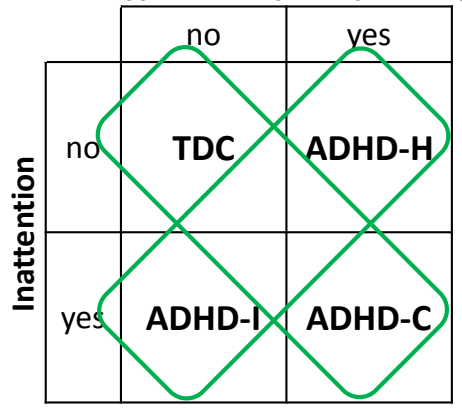

بود. لازم به ذكر است همه كودكان ADHD، ب شاعت قبل از اجراى آزمايش مصرف دارو را قطع كرده بودند. پس انتخاب گروه نمونه،

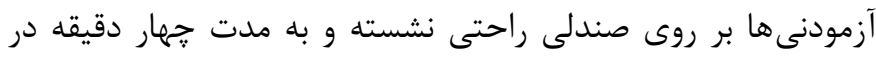
حالت جشم بسته از آنها EEG ثبت شد.

جك ليست رفتارى كودكان (Child Behavior Checklist (CBCL) اين آزمون جهت ارزيابى جامع مشكلات رفتارى عاطفى كودكان نظير: ارزيابى واكنشهاى هيجانى كودكان، ارزيابى روابط اجتماعى إنى إنى إنى إنى كودكان و سطح تعامل وى، ارزيابى دامنه فعاليتهاى بهنجار /نابهنجار كودى، ارزيابى قابليت و شايستخى كودى، اضطراب و افسردىى، مشكلات جسمانى، مشكلات اجتماعى، مشكلات تفكر، نقص توجه/

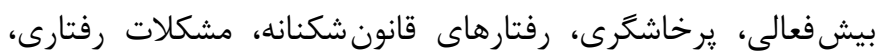

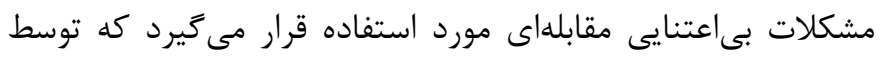
والدين يا مربيان فرد تكميل مىشود (Tr).

الكتروانسفالوكرافى كمى (Q) electroencephalography (QEEG) (Quantitative سيخنالها و امواج الكتريكى شكل گرفته توسط فعاليت مغزى مىتوانند توسط الكترودهاى متصل به يوست سر دريافت و ثبت گردند، كه اين

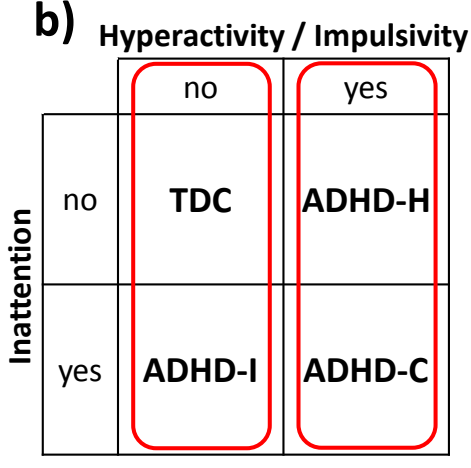

\begin{tabular}{|ll|}
\hline TDC & $\begin{array}{l}\text { ADHD Presentations } \\
\text { typically developing children }\end{array}$ \\
ADHD-H & $\begin{array}{l}\text { ADHD predominantly } \\
\text { hyperactive-impulsive }\end{array}$ \\
ADHD-I & ADHD predominantly inattentive \\
ADHD-C & ADHD combined type
\end{tabular}

statistical Tests

a) Inatt. (main effect)

b) Hyp./Imp. (main effect)

c) Inatt $\times$ Hyp./Imp (interaction effect) 
جدول ا. ويزَكى هاى جمعيتشناختى آزمودنىها

\begin{tabular}{|c|c|c|c|c|c|c|}
\hline $\mathbf{P}$ & $\mathbf{F}$ & سالم & ADHD-C & ADHD-H & ADHD-I & متغير \\
\hline- & - & kr & ra & 14 & Tr & تعداد \\
\hline .111 & $r / \cdot r$ & $q / F I(I / F r)$ & $9 / T \Delta(1 / 19)$ & $q / F(Y / \cdot 1)$ & $\Lambda / \&(I / f \Delta)$ & سن \\
\hline .190 & $\cdot / 1$ & $1 . Y / \Delta 9(1 \cdot / 9)$ & $1 . r / 9 r(11 / 1)$ & $1.9(1 T)$ & $1 \cdot r / N T(1 \cdot / 9 T)$ & هوش \\
\hline
\end{tabular}

بافتهها

درون گروهى، تفاوت معنادارى در نواحى مختلف مغزى در گروههاى ADHD-H و ADHD-I ADHD-I بود. هيج تفاوت معنادارى در توان مطلق و نسبى توان آلفا بين كروهها

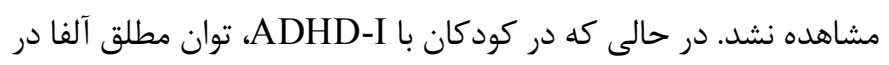

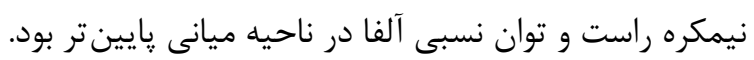

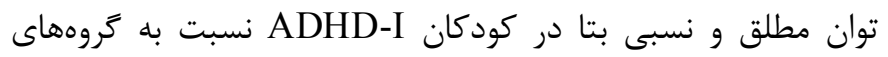
ديكر پِايينتر بود و اين گروه توان مطلق بالايى را در نواحى پس سرى و نيمكره راست نشان دادند. نسبت تتا/بتا در اثر تعاملى ADHD-I و ADHD-H ADHD-I

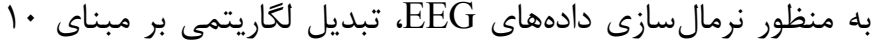
انجام شد و توان مطلق، نسبى و نسبت توان در نواحى كه به آنها اشاره شده بود در حالت جشم بسته محاسبه شدند. جدول r ميانكين و انحراف معيار را براى هر يك از گروهها ارائه مى كند. جدول ب خلاصه نتايج

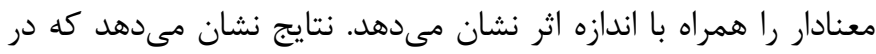
كودكان ADHD-I، توان مطلق باند دلتا در ناحيه مركزى و نيمكره راست بالاتر بود و نيز توان نسبى دلتا در ناحيه يسسرى بزر گتر بود. اما

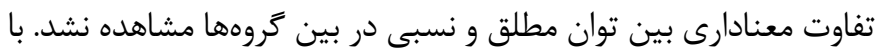
توجه به اهميت ناحيه Cz در اين گونه مطالعات، نمودار و شكل ب تفاوت كروهها را بر اساس توان مطلق تتا و بتا و نيز نسبت تتا/بتا نشان مىدهد. توان مطلق و نسبى تتا در كودكان ADHD-I بيشتر بود. در مقايسههاى

جدول ז. ميانكَين و انحراف استاندارد كَوهها بر اساس نواحى مغزى

\begin{tabular}{|c|c|c|c|c|c|c|c|}
\hline نيمكره راست & ميانى & نيمكره جِ & يس سرى & مر كزى & قدامى & كروه & توان مطلق \\
\hline$r / \Gamma(\cdot / \cdot r)$ & $r / r F(\cdot / \cdot r)$ & $r / \Gamma(\cdot / \cdot r)$ & $r / \mu \wedge(\cdot / \cdot r)$ & $r / r V(\cdot / \cdot r)$ & $r / r q(\cdot / \cdot r)$ & TDC & \multirow{4}{*}{ دلتا } \\
\hline$r / T \varphi(\cdot / \cdot 9)$ & $\Gamma / \mathcal{F} \Delta(\cdot / \cdot \Delta)$ & $r / T \wedge(\cdot / \cdot 9)$ & T/FT $(\cdot / \cdot 9)$ & $r / r q(\cdot / \cdot \Delta)$ & $r / r q(\cdot / \cdot V)$ & ADHD-H & \\
\hline$\Gamma / \Psi \wedge(\cdot / \cdot \varphi)$ & $r / r q(\cdot / \cdot r)$ & $r / \mu(\cdot / \cdot f)$ & $r / r \wedge(\cdot / \cdot \varphi)$ & $r / r q(\cdot / \cdot \varphi)$ & r/ & ADHD-I & \\
\hline$r / \mathcal{f}(\cdot / \cdot \Delta)$ & $r / r V(\cdot / \cdot \Delta)$ & $r / r \checkmark(\cdot / \cdot \Delta)$ & 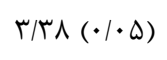 & $r / \mathcal{E} r(\cdot / \cdot \varphi)$ & ) & ADHD-C & \\
\hline$r / \cdot \varphi(\cdot / \cdot \varphi)$ & $\Gamma / 19(\cdot / \cdot r)$ & $r / \cdot V(\cdot / \cdot \varphi)$ & $r / l \tau(\cdot / \cdot \varphi)$ & $r / l r(\cdot / \cdot r)$ & $r / \cdot \Delta(\cdot / \cdot \varphi)$ & TDC & \multirow{4}{*}{ تتا } \\
\hline$\uparrow / \curlywedge \wedge(\cdot / \cdot \vee)$ & $r / l \varphi(\cdot / .9)$ & $r / \Lambda \Lambda(\cdot / \cdot \vee)$ & $r / r \Lambda(\cdot / \cdot \vee)$ & $r / l V(\cdot 1 \cdot 9)$ & $r / \cdot \varphi(\cdot / \cdot \Lambda)$ & ADHD-H & \\
\hline$r / T V(\cdot / \cdot \Delta)$ & $\Gamma / T \Delta(\cdot / \cdot \varphi)$ & $r / T F(\cdot / \cdot \Delta)$ & ( & $r / r F(\cdot / \cdot F)$ & $r / 11(\cdot 1.9)$ & ADHD-I & \\
\hline$r / \mu(\cdot / \cdot 9)$ & 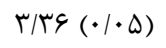 & $\Gamma / T \wedge(\cdot / \cdot \Delta)$ & $r / \mu(\cdot 1 \cdot 9)$ & 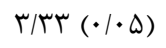 & $\pi / r r(\cdot 1 \cdot 9)$ & ADHD-C & \\
\hline
\end{tabular}


ادامه جدول r. ميانگين و انحراف استاندارد گروهها بر اساس نواحى مغزى

\begin{tabular}{|c|c|c|c|c|c|c|c|}
\hline نيمكره راست & ميانى & نيمكره جِ & يس سرى & مركزى & قدامى & كروه & توان مطلق \\
\hline r/MF $(\cdot / \cdot r)$ & $r / F \Delta(\cdot / \cdot r)$ & $r / r \Delta(\cdot / \cdot \varphi)$ & (ז) & $r / r q(\cdot / \cdot r)$ & $r / r q(\cdot / \cdot q)$ & $\mathrm{TDC}$ & \multirow{4}{*}{ آلفا } \\
\hline$r / T V(\cdot / \cdot 4)$ & $r / \mathcal{F}(\cdot / \cdot q)$ & $r / r q(\cdot / \cdot V)$ & 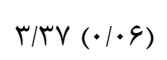 & س & $r / r q(\cdot / \cdot V)$ & ADHD-H & \\
\hline$r / \mu \mathcal{F}(\cdot / \cdot \Delta)$ & $r / r F(\cdot / \cdot F)$ & $r / r q(\cdot / \cdot \Delta)$ & 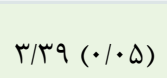 & ז/ץ $(\cdot / \cdot \Delta)$ & r/r $(\cdot \mid \cdot \Delta)$ & ADHD-I & \\
\hline$r / f \mid(\cdot / \cdot \Delta)$ & $r / \varphi q(\cdot / \cdot \varphi)$ & $r / \varphi l(\cdot / \cdot \Delta)$ & r/Dr & ז//q $(\cdot / \cdot \Delta)$ & $r / r \Delta(\cdot / \cdot 4)$ & ADHD-C & \\
\hline$r / l f(\cdot / \cdot \varphi)$ & $r / \Lambda \Lambda(\cdot / \cdot r)$ & $r / \backslash \Lambda(\cdot / \cdot \varphi)$ & T/TT $(\cdot / \cdot F)$ & 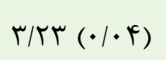 & $r / \cdot q(\cdot / \cdot q)$ & TDC & \multirow{4}{*}{ بتا } \\
\hline$r / \cdot r(\cdot / \cdot \Lambda)$ & $r / 1)(\cdot / \cdot \Lambda)$ & $r / l r(\cdot / \cdot V)$ & $\Gamma / / \Delta(\cdot / \cdot \Lambda)$ & $r / 1 \varepsilon(\cdot / \cdot V)$ & $r / q F(\cdot / \cdot \Lambda)$ & ADHD-H & \\
\hline$\Gamma / 9 \Delta(\cdot / \cdot 4)$ & $r / \wedge 9(\cdot / \cdot 4)$ & r/qT $(\cdot / \cdot \Delta)$ & $r / 9 \Lambda(\cdot / \cdot 9)$ & $\operatorname{r/Ar}(\cdot / \cdot \Delta)$ & $r / 9 \Delta(\cdot / \cdot 9)$ & ADHD-I & \\
\hline $\mathrm{r} / \Lambda V(\cdot / \cdot 4)$ & $r / A r(\cdot / \cdot 9)$ & r/Ar $(\cdot / \cdot 9)$ & $r / 9 F(\cdot 1.4)$ & T/Vq $(\cdot / \cdot 9)$ & $\operatorname{r/V} q(\cdot / \cdot 9)$ & ADHD-C & \\
\hline $.199(\cdot / \cdot r)$ & $1(\cdot / \cdot 1)$ &.$/ 91(\cdot / \cdot 1)$ &.$/ 91(\cdot / \cdot 1)$ &.$/ 9 \vee(\cdot / \cdot 1)$ & $1 / \cdot 1(\cdot / \cdot r)$ & TDC & \multirow{4}{*}{ سبت تتا/بتا } \\
\hline $1 / \cdot \varphi(\cdot / \cdot r)$ & $1 / \cdot r(\cdot / \cdot r)$ & $1 / \cdot r(\cdot / \cdot r)$ & $1 / \cdot \Delta(\cdot / \cdot r)$ & $1 / \cdot 1(\cdot / \cdot r)$ & $1 / \cdot \Delta(\cdot / \cdot r)$ & ADHD-H & \\
\hline $1 / 11(\cdot / \cdot r)$ & $1 / 1 r(\cdot / \cdot r)$ & $1 / 1 r(\cdot / \cdot r)$ & $1 / 1 r(\cdot / \cdot r)$ & $1 / 1 \wedge(\cdot / \cdot r)$ & $1 / \cdot 4(\cdot / \cdot r)$ & ADHD-I & \\
\hline $1 / \cdot r(\cdot / \cdot r)$ & $1 / r(\cdot / \cdot r)$ & $1 / 1 V(\cdot / \cdot r)$ & $1 / 1 r(\cdot / \cdot r)$ & $1 / \cdot r(\cdot / \cdot r)$ & $1 / r(\cdot / \cdot r)$ & ADHD-C & \\
\hline
\end{tabular}

Typically Developing Children (TDC), Attention Deficit Hyperactivity Disorder-Inattentive (ADHD-I), Attention Deficit Hyperactivity Disorder- Im(pulsive/hyperactive (ADHD-H), Attention Deficit Hyperactivity Disorder-Combined (ADHD-C

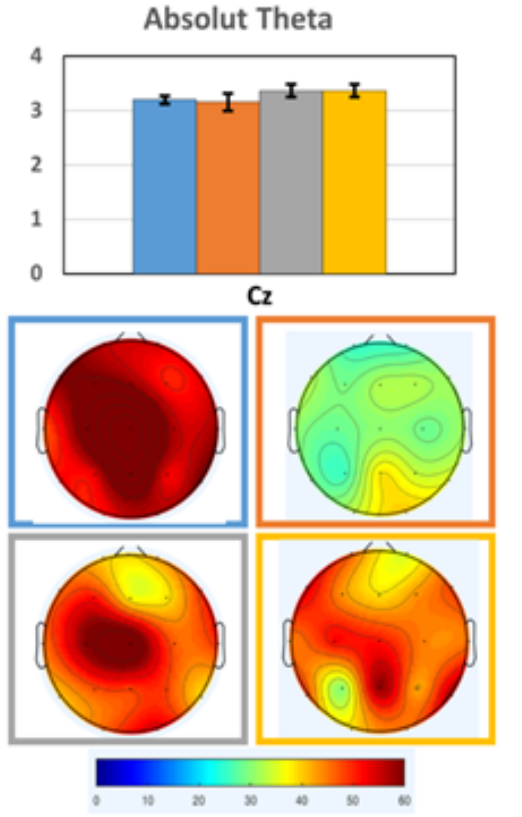

Controls

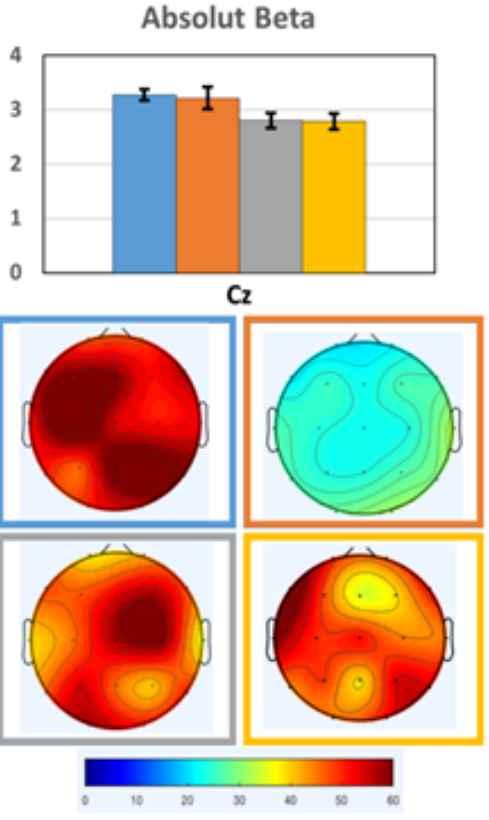

ADHD-I

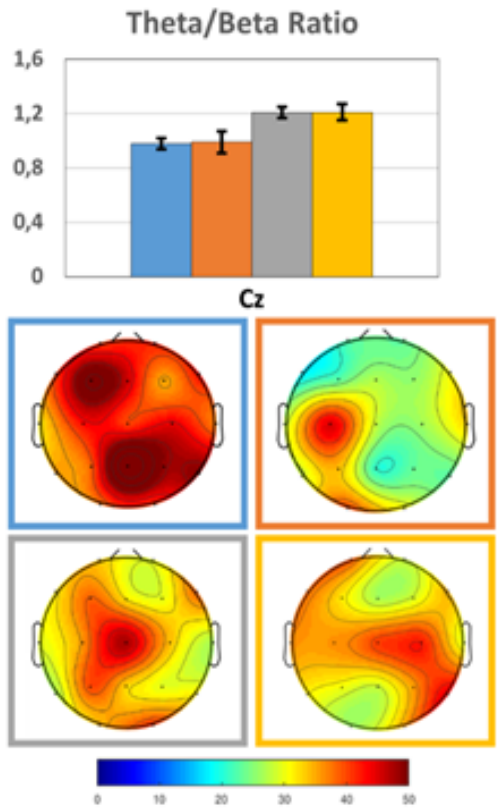

ADHD-C

شكل r. ميانگين و فاصله اطمينان توان مطلق باندهاى فر كانسى به تفكيك گروه 
جدول ؟ّ. ميانكَين و انحراف استاندارد كَروها بر اساس نواحى مغزى

\begin{tabular}{|c|c|c|c|c|c|c|}
\hline نسبت توان & \multicolumn{4}{|c|}{ توان مطلق } & \multicolumn{2}{|c|}{ مقايسه } \\
\hline 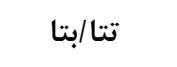 & بتا & آلفا آلفا & تتا & 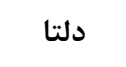 & تروهها & تفاوت هاى كروهى \\
\hline $\begin{array}{c}Y / / T D^{m s} \\
{[\cdot / \Gamma]}\end{array}$ & 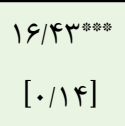 & $\begin{array}{c}\cdot / 1 \\
{[<\cdot / \cdot 1]}\end{array}$ & $\begin{array}{l}9 / \wedge V^{* \circ} \\
{[\cdot / \cdot 9]}\end{array}$ & $\begin{array}{c}\cdot 109 \\
{[<\cdot / \cdot 1]}\end{array}$ & ADHD-I & \\
\hline $\begin{array}{l}9 / \Lambda^{* * *} \\
{[\cdot / \cdot 1]}\end{array}$ & $\begin{array}{l}\text { l/Ar } \\
{[\cdot / \cdot r]}\end{array}$ & $\begin{array}{c}\cdot / \cdot r \\
{[<\cdot / \cdot 1]}\end{array}$ & $\begin{array}{c}1 / V \\
{[\cdot / \cdot r]}\end{array}$ & $\begin{array}{c}\cdot / 19 \\
{[<\cdot / \cdot 1]}\end{array}$ & ADHD-H & اثرات اصلى \\
\hline $\begin{array}{c}\cdot / \Delta \\
{[<\cdot / \cdot 1]}\end{array}$ & $\begin{array}{l}<\cdot / \cdot 1 \\
{[<\cdot / \cdot 1]}\end{array}$ & $\begin{array}{l}1 / 99 \\
{[\cdot / \cdot r]}\end{array}$ & $\begin{array}{l}<\cdot / \cdot 1 \\
{[<\cdot / \cdot 1]}\end{array}$ & $\begin{array}{c}\cdot / r r \\
{[<\cdot / \cdot 1]}\end{array}$ & ADHD-I $\times$ ADHD-H & \\
\hline 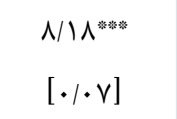 & $\begin{array}{l}\mid Q / \cdot \Delta^{\text {swas }} \\
{[\cdot / \mid \psi]}\end{array}$ & $\begin{array}{l}1 / \pi \wedge \\
{[\cdot / \cdot 1]}\end{array}$ & $\begin{array}{l}1 / 1 r \\
{[\cdot / \cdot 1]}\end{array}$ & $\begin{array}{l}41 \cdot r^{* * * a} \\
{[\cdot 1 \cdot 9]}\end{array}$ & ADHD-I & \\
\hline $\begin{array}{l}{[/ \Delta} \\
r / \cdot \cdot r]\end{array}$ & $\begin{array}{l}1 / \Delta \\
{[\cdot / \cdot 1]}\end{array}$ & $\begin{array}{l}{\left[\begin{array}{l}0 \\
{[\cdot / \cdot r]}\end{array}\right]} \\
r / 4 q\end{array}$ & $\begin{array}{l}1 / \cdot r \\
{[\cdot / \cdot 1]}\end{array}$ & $\begin{array}{c}\cdot|\Delta| \\
{[<\cdot|\cdot|]}\end{array}$ & ADHD-H & قدامى / قس سرى \\
\hline $\begin{array}{l}\Delta / \mathcal{F} V^{* * \omega} \\
{[\cdot / \cdot \Delta]}\end{array}$ & $\begin{array}{c}\cdot / \mu q \\
{[<\cdot / \cdot 1]}\end{array}$ & $\begin{array}{c}\cdot / F^{F} \\
{[<\cdot / \cdot 1]}\end{array}$ & $\begin{array}{l}9 / 9 Y^{\text {was }} \\
{[\cdot / \cdot 9]}\end{array}$ & $\begin{array}{c}\cdot / \cdot \Delta \\
{[<\cdot / \cdot 1]}\end{array}$ & ADHD-I $\times$ ADHD-H & \\
\hline $\begin{array}{c}r \\
{[\cdot / \cdot r]}\end{array}$ & $\begin{array}{l}9 / \pi 9^{* *} \\
{[\cdot 1 \cdot 9]}\end{array}$ & $\begin{array}{l}r / 1 / * \\
{[\cdot / \cdot \%]}\end{array}$ & $\begin{array}{c}\cdot / 19 \\
{[<\cdot / \cdot 1]}\end{array}$ & $\begin{array}{l}\Delta / Q^{* * a} \\
{[\cdot / \cdot \Delta]}\end{array}$ & ADHD-I & \\
\hline $\begin{array}{c}\cdot / \mu r \\
{[<\cdot / \cdot 1]}\end{array}$ & $\begin{array}{c}\cdot / \mu q \\
{[<\cdot / \cdot 1]}\end{array}$ & $\begin{array}{c}\cdot \mid \Delta F \\
{[<\cdot / \cdot 1]}\end{array}$ & $\begin{array}{c}\cdot / F^{f} \\
{[<\cdot / \cdot 1]}\end{array}$ & $\begin{array}{c}r / \mathcal{F} \\
{[\cdot / \cdot r]}\end{array}$ & ADHD-H & جِ|راست \\
\hline $\begin{array}{c}{[\cdot / \cdot r]} \\
r\end{array}$ & $\begin{array}{c}\cdot / 4 \\
{[<\cdot / \cdot 1]}\end{array}$ & $\begin{array}{c}\cdot / r V \\
{[<\cdot / \cdot 1]}\end{array}$ & $\begin{array}{l}{\left[/ r r^{*}\right.} \\
{[\cdot / \cdot r]}\end{array}$ & $\begin{array}{c}\cdot 199 \\
{[<\cdot / \cdot 1]}\end{array}$ & ADHD-I $\times$ ADHD-H & \\
\hline $\begin{array}{c}1 \\
{[<\cdot / \cdot 1]}\end{array}$ & $\begin{array}{c}\cdot / 9 \\
{[<\cdot / \cdot 1]}\end{array}$ & $\begin{array}{c}\cdot / v 9 \\
{[<\cdot / \cdot 1]}\end{array}$ & $\begin{array}{c}\cdot / 9 \mu \\
{[<\cdot / \cdot 1]}\end{array}$ & $\begin{array}{l}r / 9 Y^{\circ} \\
{[\cdot / \cdot r]}\end{array}$ & ADHD-I & \\
\hline $\begin{array}{l}1 / / 49 \\
{[\cdot / \cdot 1]}\end{array}$ & $\begin{array}{c}\cdot / V \\
{[<\cdot / \cdot 1]}\end{array}$ & $\begin{array}{c}\cdot / \cdot r \\
{[<\cdot / \cdot 1]}\end{array}$ & $\begin{array}{c}\cdot / r r \\
{[<\cdot / \cdot 1]}\end{array}$ & $\begin{array}{c}\cdot / A r \\
{[<\cdot / \cdot \mid]}\end{array}$ & ADHD-H & 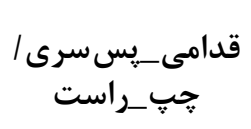 \\
\hline $\begin{array}{c}\cdot / \wedge \Delta \\
{[<\cdot / \cdot 1]}\end{array}$ & $\begin{array}{c}\cdot / r \\
{[<\cdot / \cdot 1]}\end{array}$ & $\begin{array}{l}1 / \cdot 1 \\
{[\cdot / \cdot 1]}\end{array}$ & $\begin{array}{l}1 / 4 F \\
{[\cdot / \cdot 1]}\end{array}$ & $\begin{array}{l}1 / \mathrm{A \Lambda} \\
{[\cdot / \cdot r]}\end{array}$ & ADHD-I × ADHD-H & \\
\hline
\end{tabular}

${ }^{\circ} \mathrm{P}<\cdot 1 \cdot \Delta,{ }^{\circ} \mathrm{P}<\cdot|\cdot|,,{ }^{\circ * m} \mathrm{P}<\cdot \mid \cdot \cdot 1$

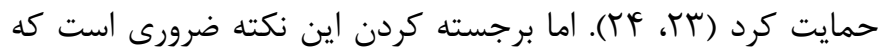

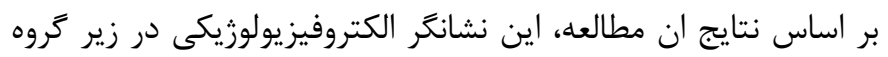

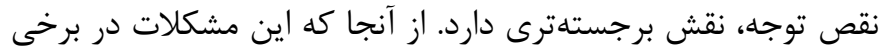

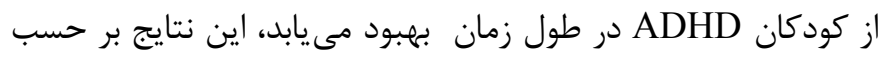
مدل تاخير بلوغ سيستم عصبى مركزى قابل تبيين مىشود. بر اساس

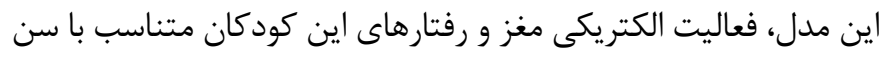

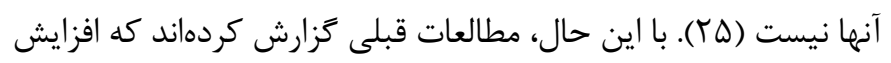

نسبت تتابتا در همه زير گروههاى ADHD به ويزه زير گروه ADHD-I در مقايسه با كودكان عادى بالاتر بود. به علاوه در زير تروه ADHD-I، فعاليت تتا بالاتر و فعاليت بتا پايينتر بود. هيج تفاوت معنادارى بين كروهها در فعاليت دلتا و آلفا مشاهده نشد. براى هر سه يارامتر EEG (يعنى تتا، بتا و نسبت تتا/بتا)، تنها عامل نقص توجه تاثير داشت. يافتههاى مطالعه

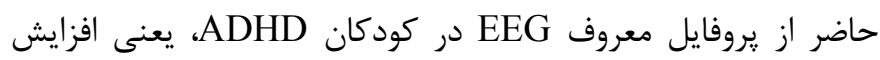

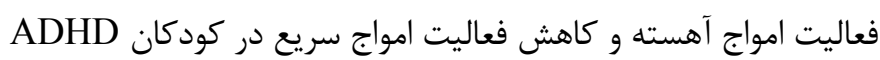


به طور خلاصه، تفاوت در فعاليت EEG در گروهها با افزايش توان

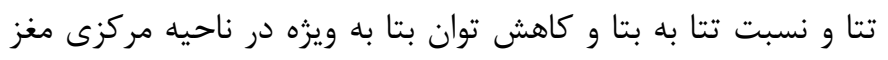

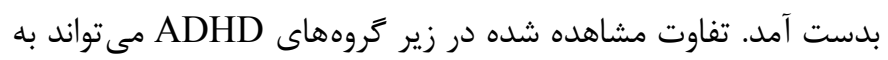

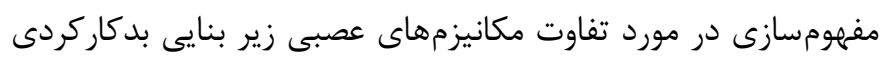

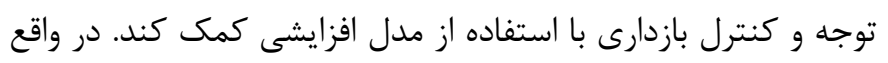

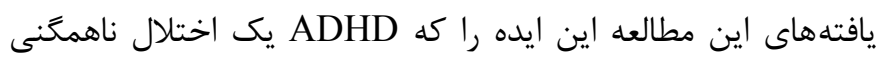

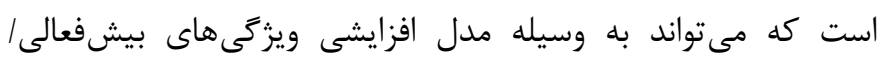
تكانشخرى و نقص توجه در زير گروههاى قابل تفكيك تقسيم شوده،

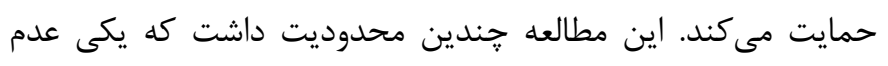

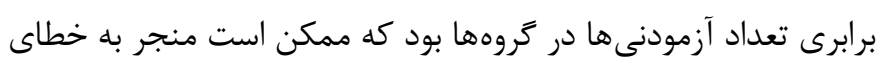

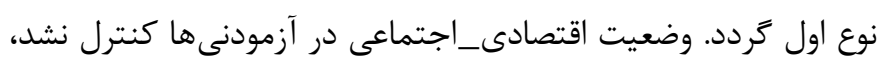

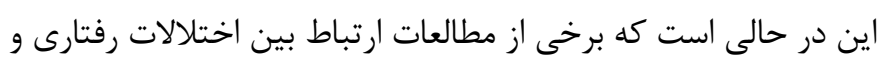

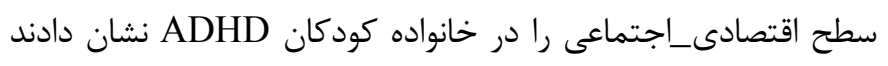

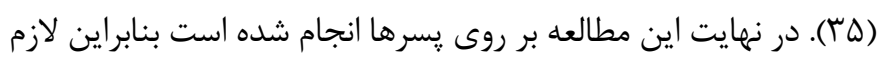
است تعميم نتايج به دختران مبتلا به ADHD با احتياط انجام كردد.

\section{نتيجه كيرى}

بر اساس نتايج اين مطالعه، نشانكر هاى الكتروانسفالوكر افى زير كروه نقص

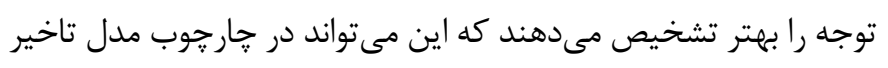

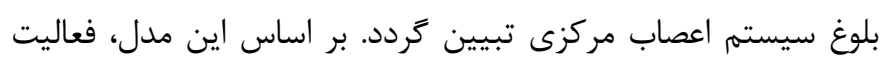

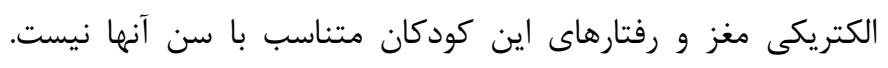

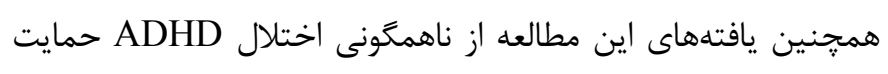

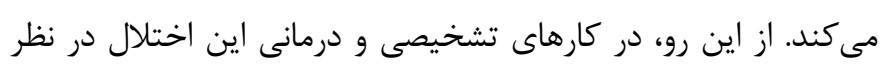
كرفتن ناهمًونى اين اختلال توصيه مى كردد.

\section{تشكر و قدرهانى}

بدينوسيله از آقايان دكتر رضا رستمى و دكتر مسعود نصرت آبادى كه

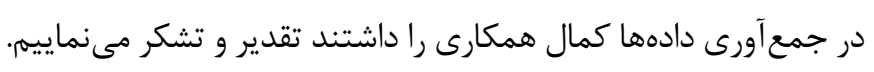

\section{References}

1. American Psychiatric Association. Diagnostic and statistical manual of mental disorders (DSM-5®). Arlington VA:American Psychiatric Association;2013.

2. Moayedi F, Moayedi A, Goli G, Hamedi Y. Prevalence of attention deficit hyperactivity disorder in Bandarabbas pri-
در فعاليت تتا يك نشانكر عصبى غير اختصاصى نابهنجار براى ADHD

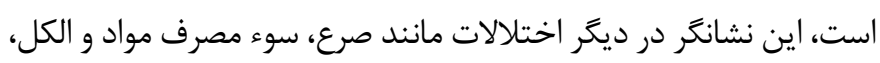

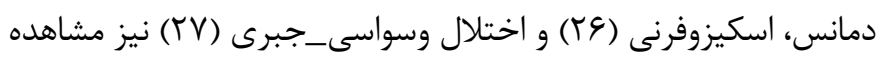

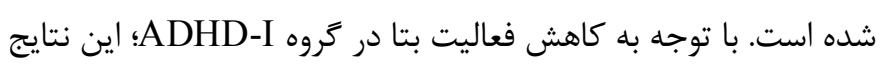

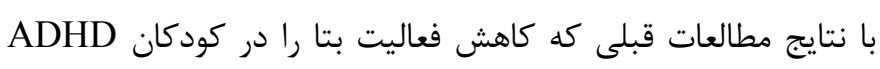

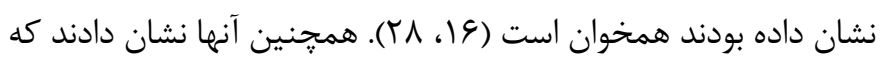

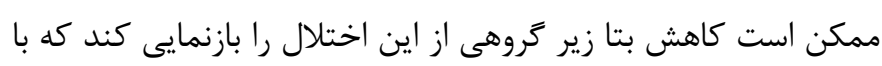

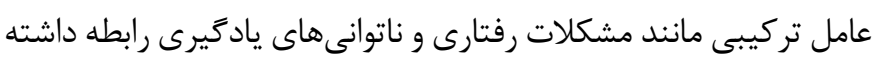

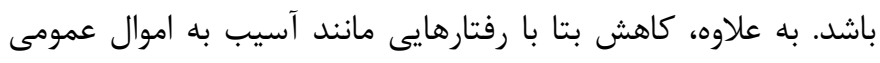
(Vandalism)، نوسان خلق و رفتارهاى ضد اجتماعى رابطه دارد (9 (Y). در كل، كاهش بتا با آسيب كاركردهاى شناختى در كودكان ADHD

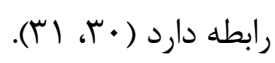

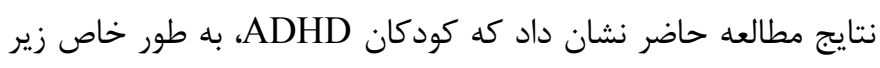

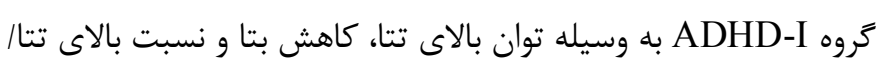

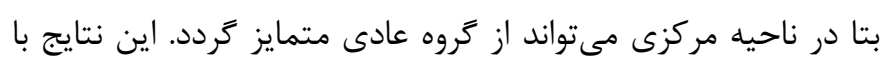

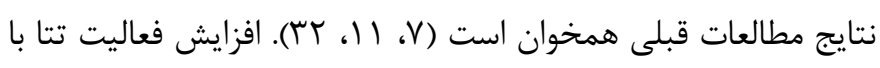

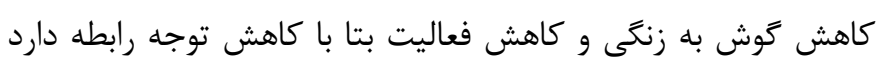

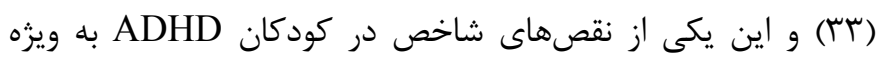

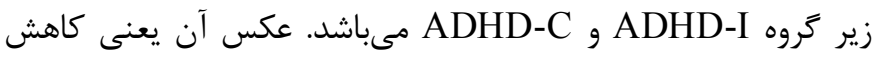

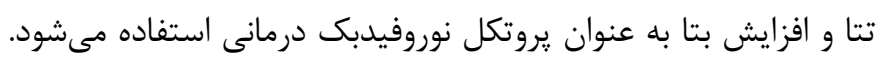

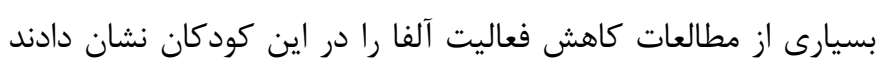

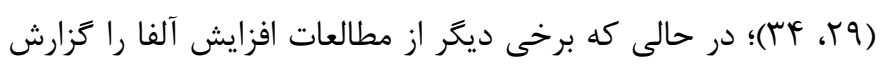

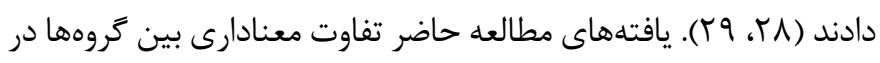

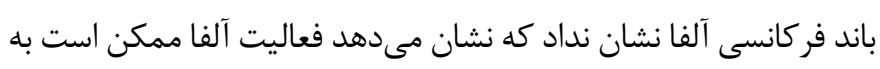
تنهايى نشان دهنده بعضى از ناهمخنى ها در

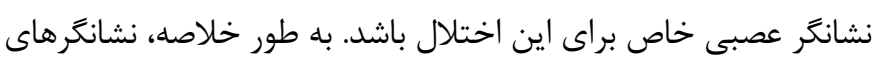

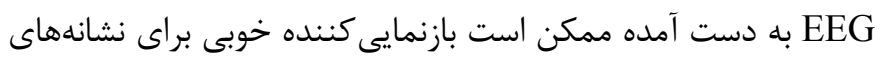
ADHD

mary school students in 2010. Hormozgan Medical Journal. 2011;17(3):241-247. (Persian)

3. Milich R, Balentine AC, Lynam DR. ADHD combined type and ADHD predominantly inattentive type are distinct and unrelated pdisorders. Clinical Psychology: Science and Practice. 
2001;8(4):463-488.

4. Baeyens D, Roeyers H, Walle JV. Subtypes of attention-deficit/hyperactivity disorder (ADHD): Distinct or related disorders across measurement levels?. Child Psychiatry and Human Development. 2006;36(4):403-417.

5. Jeste SS, Nelson CA. Event related potentials in the understanding of autism spectrum disorders: An analytical review. Journal of Autism and Developmental Disorders. 2009;39(3):495-510.

6. Kang JQ, Barnes G. A common susceptibility factor of both autism and epilepsy: Functional deficiency of GABA A receptors. Journal of Autism and Developmental Disorders. 2013;43(1):68-79.

7. Lubar JF. Discourse on the development of EEG diagnostics and biofeedback for attention-deficit/hyperactivity disorders. Biofeedback and Self-regulation. 1991;16(3):201-225.

8. Snyder SM, Quintana H, Sexson SB, Knott P, Haque AF, Reynolds DA. Blinded, multi-center validation of EEG and rating scales in identifying $\mathrm{ADHD}$ within a clinical sample. Psychiatry Research. 2008;159(3):346-358.

9. Jeste SS, Frohlich J, Loo SK. Electrophysiological biomarkers of diagnosis and outcome in neurodevelopmental disorders. Current Opinion in Neurology. 2015;28(2):110-116.

10. Van Dongen-Boomsma M, Lansbergen MM, Bekker EM, Kooij JS, Van Der Molen M, Kenemans JL, et al. Relation between resting EEG to cognitive performance and clinical symptoms in adults with attention-deficit/hyperactivity disorder. Neuroscience Letters. 2010;469(1):102-106.

11. Williams LM, Hermens DF, Thein T, Clark CR, Cooper NJ, Clarke SD, et al. Using brain-based cognitive measures to support clinical decisions in ADHD. Pediatric Neurology. 2010;42(2):118-126.

12. Nazari MA, Wallois F, Aarabi A, Berquin P. Dynamic changes in quantitative electroencephalogram during continuous performance test in children with attention-deficit/hyperactivity disorder. International Journal of Psychophysiology. $2011 ; 81(3): 230-236$.
13. Ogrim G, Hestad KA. Effects of neurofeedback versus stimulant medication in attention-deficit/hyperactivity disorder: A randomized pilot study. Journal of Child and Adolescent Psychopharmacology. 2013;23(7):448-457.

14. Liechti MD, Valko L, Müller UC, Döhnert M, Drechsler R, Steinhausen HC, et al. Diagnostic value of resting electroencephalogram in attention-deficit/hyperactivity disorder across the lifespan. Brain Topography. 2013;26(1):135-151.

15. Arns M, Conners CK, Kraemer HC. A decade of EEG theta/ beta ratio research in ADHD: A meta-analysis. Journal of Attention Disorders. 2013;17(5):374-483.

16. Clarke AR, Barry RJ, McCarthy R, Selikowitz M. Age and sex effects in the EEG: Differences in two subtypes of attention-deficit/hyperactivity disorder. Clinical Neurophysiology. 2001;112(5):815-826.

17. Loo SK, Makeig S. Clinical utility of EEG in attention-deficit/hyperactivity disorder: A research update. Neurotherapeutics. 2012;9(3):569-587.

18. Gloss D, Varma JK, Pringsheim T, Nuwer MR. Practice advisory: The utility of EEG theta/beta power ratio in ADHD diagnosis: Report of the guideline development, dissemination, and implementation subcommittee of the American Academy of Neurology. Neurology. 2016;87(22):2375-2379.

19. Mazaheri A, Fassbender C, Coffey-Corina S, Hartanto TA, Schweitzer JB, Mangun GR. Differential oscillatory electroencephalogram between attention-deficit/hyperactivity disorder subtypes and typically developing adolescents. Biological Psychiatry. 2014;76(5):422-429.

20. Ahmadi N, Mohammadi MR, Araghi SM, Zarafshan H. Neurocognitive profile of children with attention deficit hyperactivity disorders (ADHD): A comparison between subtypes. Iranian Journal of Psychiatry. 2014;9(4):197-202.

21. Insel T, Cuthbert B, Garvey M, Heinssen R, Pine DS, Quinn K, et al. Research domain criteria (RDoC): Toward a new classification framework for research on mental disorders. The American Journal of Psychiatry. 2010;167(7):748-751. 22. Achenbach TM, Rescorla LA. Manual for the ASEBA 
school-age forms \& profiles. Burlingron, VT:University of Vermont, Research Center of Children, Youth \& Families;2001.

23. Aldemir R, Demirci E, Per H, Canpolat M, Ozmen S, Tokmakci M. Investigation of attention deficit hyperactivity disorder (ADHD) sub-types in children via EEG frequency domain analysis. International Journal of Neuroscience. 2018;128(4):349-360.

24. Heinrich H, Busch K, Studer P, Erbe K, Moll GH, Kratz O. EEG spectral analysis of attention in ADHD: Implications for neurofeedback training?. Frontiers in Human Neuroscience. 2014;8:611.

25. Markovska-Simoska S, Pop-Jordanova N. Quantitative EEG in children and adults with attention deficit hyperactivity disorder: Comparison of absolute and relative power spectra and theta/beta ratio. Clinical EEG and Neuroscience. 2017;48(1):20-32.

26. Coutin-Churchman P, Anez Y, Uzcategui M, Alvarez L, Vergara F, Mendez L, et al. Quantitative spectral analysis of EEG in psychiatry revisited: Drawing signs out of numbers in a clinical setting. Clinical Neurophysiology. 2003;114(12):2294-2306.

27. Budzynski TH, Budzynski HK, Evans JR, Abarbanel A. Introduction to quantitative EEG and neurofeedback: Advanced theory and applications. 2nd ed. Cambridge:Academic Press;2009

28. Chabot RJ, Serfontein G. Quantitative electroencephalographic profiles of children with attention deficit disorder. Biological Psychiatry. 1996;40(10):951-963.

29. Clarke AR, Barry RJ, Dupuy FE, Heckel LD, McCarthy R,
Selikowitz M, et al. Behavioural differences between EEG-defined subgroups of children with attention-deficit/hyperactivity disorder. Clinical Neurophysiology. 2011;122(7):1333-1341. 30. Hermens DF, Soei EX, Clarke SD, Kohn MR, Gordon E, Williams LM. Resting EEG theta activity predicts cognitive performance in attention-deficit hyperactivity disorder. Pediatric Neurology. 2005;32(4):248-256.

31. Sumich A, Matsudaira T, Gow RV, Ibrahimovic A, Ghebremeskel K, Crawford M, et al. Resting state electroencephalographic correlates with red cell long-chain fatty acids, memory performance and age in adolescent boys with attention deficit hyperactivity disorder. Neuropharmacology. 2009;57(78):708-714.

32. Snyder SM, Rugino TA, Hornig M, Stein MA. Integration of an EEG biomarker with a clinician's ADHD evaluation. Brain and Behavior. 2015;5(4):e00330.

33. Banaschewski T, Brandeis D. Annotation: What electrical brain activity tells us about brain function that other techniques cannot tell us-a child psychiatric perspective?. Journal of child Psychology and Psychiatry. 2007;48(5):415-435.

34. Buyck I, Wiersema JR. Electroencephalographic activity before and after cognitive effort in children with attention deficit/hyperactivity disorder. Clinical EEG and Neuroscience. 2015;46(2):88-93.

35. Kadesjo C, Hägglof B, Kadesjo B, Gillberg C. Attention-deficit-hyperactivity disorder with and without oppositional defiant disorder in 3-to 7-year-old children. Developmental Medicine and Child Neurology. 2003;45(10):693-699. 